\title{
Individual Energetics and the Equilibrium Demography of Structured Populations
}

\author{
William S. C. Gurney* and David A. J. Middleton \\ Department of Statistics \& Modelling Science, University of Strathclyde, \\ Glasgow G1 $1 \mathrm{XH}$, Scotland
}

ROGER M. NisBet

Department of Biological Sciences, University of California, Santa Barbara, California 93106

EDWARD MCCAULEY

Department of Biological Sciences, University of Calgary, Calgary, Alberta, Canada T2N 1 N4

William W. MuRdoch

Department of Biological Sciences, University of California, Santa Barbara, California 93106

AND

ANDRE DeRoos

Department of Pure \& Applied Ecology, University of Amsterdam, Kruislaan 320, 1098 SM, Amsterdam, the Netherlands

Received December 27, 1994

This paper considers the relationship between the demographic mechanisms of population control and the energetics of the individuals who comprise the population. We examine the equilibrium properties of a class of structured population models in which individuals compete for some environmental resource (such as food) and demonstrate that population demography is independent of the nature of the feedback loop which establishes the equilibrium state. We thus derive general insights into the influence exerted by individual energetic and allocation strategies on population average demographic characteristics. We show that models of individual energetics which produce apparently very similar predictions at the individual level can result in very different behaviour at the population level. In addition, we

* To whom correspondence should be addressed. 
observe that different models of individual mortality can imply marked differences in population demography and that the common assumption of constant mortality can be responsible for potentially unrealistic model behaviour. Our results emphasise the substantial data requirements for parameterising and testing individual-based models.

(C) 1996 Academic Press, Inc.

\section{INTRODUCTION}

In recent years, steadily increasing use has been made of individualbased approaches to both population and community modelling. A wide variety of formalisms have been used, ranging from distribution-function methods (Metz and Diekmann, 1986) to individual-by-individual simulations (e.g., MacKay, 1992; Huston, 1992). However, all these disparate projects have ultimately been motivated by the view that the dynamics of a population are the product of the dynamics of the collection of individuals of which it is composed.

Individual-based models can sometimes be successfully constructed on the basis of an empirical characterisation of the demographic contribution of each constituent individual. However, this approach is most successful when the organisms concerned are sufficiently simple for a wide range of environmental conditions to be duplicated in the laboratory (e.g., phytoplankton) or when the population is composed of large individuals whose properties can be measured in situ (e.g., a forest stand).

An alternative and more generally applicable approach is to model the mechanisms which underlie the demographic performance of individuals. Growth, survival, and reproduction all depend directly or indirectly on the aquisition and utilisation of energy and nutrients. A model of an individual's contribution to population demography (hereafter refered to as individual demography) thus has two key components; first, a relationship between the physiological state of the individual (age, length, weight, reserves, etc.) and the rate at which it can acquire resources from its environment: second, a description of the way in which ingested resource is partitioned among maintenance, growth, and reproduction.

Before using any model of individual demography as the basis of a population model it is clearly prudent to test its predictions against appropriate observations. Several workers; (e.g., Kooijman, 1986, 1993; Gurney et al, 1990, Ross and Nisbet, 1990; Broekhuizen et al., 1994) have succeeded in reproducing sets of laboratory, aquaculture, and field data on the growth and reproduction of individuals of different species. Less effort has been devoted to the development of energetically based models of mortality (notable exceptions being Anderson, 1992; Kooijman, 1993, pp. 105-112), partly perhaps because of the relative paucity of data for testing, but also because many of the key components of mortality 
(predation, parasitism, etc.) are only very indirectly related to energetic considerations.

Even in the relatively well-studied area of growth and reproduction, testing an individual model requires a very large quantity of data. For example, the length at age curves predicted under constant food conditions by the Kooijman-Metz allocation model (Kooijman, 1986) are identical to those predicted under the (apparently minor) extension proposed by Thieme (1988). Although the two models predict fecundity at age curves which can certainly be distinguished by careful examination of the pattern of fecundity variation near the onset of reproduction, the differences are subtle enough to be easily missed unless the experimental protocol is specifically planned to reveal them.

Given the difficulty of distinguishing among a group of plausible hypotheses about individual energy allocation even on the basis of apparently good data about individual demography it becomes particularly important to know whether these subtleties matter at the population level. Suppose that a consumer population, at equilibrium, is controlled by the availability of a single resource with no other direct or indirect density dependent processes operating. The demographic properties of the individual organisms (growth, fecundity, and mortality rates) will all be set by resource availability, which is itself controlled by balance between supply (or renewal) and uptake by the consumer population. Thus, at equilibrium, the demographic properties of individuals must be strongly interdependent. For example, if the (resource independent) environmental component of mortality increases, equilibrium can be maintained only by a compensatory change in growth rate, fecundity, or the density-dependent component of mortality. Such compensation can occur as a result of an increase in the equilibrium level of resource availablity, which must necessarily affect all of the demographic characteristics of the individuals in the population.

The implication of this interrelationship is that the details of the way in which individuals allocate energy may be linked in subtle ways to changes in the demographic mechanisms by which population control is expressed. In this paper we explore this relationship, and, in particular, we identify circumstances in which a single resource-dependent demographic process will appear to be the sole agent of population control. Our primary methodology, discussed at more length in the section entitled "observable demographic characteristics," is to explore the sensitivity of demographic properties, which might be expected to be observable in the field, to changes in the background (environmental) component of mortality. We discuss the implications of our results for real populations, particularly those for which some characteristics (for example juvenile development time) are subject to physiological constraints. 


\section{THEORETICAL FRAMEWORK}

The equilibrium age-distribution. We consider a population within which individuals are distinguished by two physiological attributes: age $(a)$, and an as-yet undefined index of development which we shall denote by $q$. We assume that the demographic characteristics of each individual are determined by its physiological state $(a, q)$ and the effects of its environment. Provided that the population is well enough mixed for us to safely assume that all individuals experience the same environmental conditions $(\psi)$, we can write the per capita mortality, fecundity, and development rate functions as $\delta(a, q, \psi), \beta(a, q, \psi)$, and $g(a, q, \psi)$, respectively.

The formulation of models of such populations has been extensively discussed by Metz and Diekmann (1986), who show that if all individuals enter the population with the same development index $\left(q_{R}\right)$ and experience the same environmental conditions, then all the members of any given cohort must have equal development indexes throughout their lives. This implies that the equilibrium state of such a population can conveniently be described by the combination of an equilibrium number-at-age distribution, $f(a)$, defined so that $f(a) d a$ represents the number of individuals in the equilibrium population whose age lies in the range $a$ to $a+d a$, and a development-at-age function, $\theta(a)$, given by

$$
\theta(a)=q_{R}+\int_{0}^{a} g(x, \theta(x), \psi) d x
$$

The shape of the equilibrium number-at-age distribution is related to $S(a, \psi)$, the survival from recruitment to age a in constant environmental conditions $\psi$, by

$$
f(a)=f(0) S(a, \psi)
$$

where

$$
S(a, \psi)=\exp \left[-\int_{0}^{a} \delta(x, \theta(x), \psi) d x\right] .
$$

The equilibrium value of the environment parameter $\left(\psi^{*}\right)$ is determined by the renewal condition, which requires that the density of zero-age individuals be equal to the rate of production of new offspring, that is,

$$
f(0)=\int_{0}^{\infty} f(x) \beta\left(x, \theta(x), \psi^{*}\right) d x .
$$


Substituting Eq. (2a) into Eq. (3a) and then cancelling the factor of $f(0)$ which appears on both sides of the result yields the characteristic equation (Gurtin and McCamy, 1981)

$$
\int_{0}^{\infty} S\left(x, \psi^{*}\right) \beta\left(x, \theta(x), \psi^{*}\right) d x=1
$$

which can be solved for $\psi^{*}$.

Equations (1), (2), and (3) determine the shape of the equilibrium number-at-age distribution $\left(S\left(a, \psi^{*}\right)\right)$ but not its absolute size (i.e., $f(0)$ ). In addition to the foregoing equations, a complete model of a controlled population must contain a description of some feedback loop which controls the value of the environment parameter in response to the age and development distributions. Consider, for example, a case in which the appropriate choice of environment variable is the density of available resources. If new resource is introduced into the system at a rate $P(\psi)$, and an individual in physiological state $(a, q)$ consumes resource at a rate $U(a, q, \psi)$, then the equilibrium state requires equality of total production and total consumption rates, that is,

$$
P\left(\psi^{*}\right)=\int_{0}^{\infty} f(x) U\left(x, \theta(x), \psi^{*}\right) d x
$$

Substituting from Eq. (2a) yields

$$
f(0)=\frac{P\left(\psi^{*}\right)}{\int_{0}^{\infty} S\left(x, \psi^{*}\right) U\left(x, \theta(x), \psi^{*}\right) d x} .
$$

The equilibrium development distribution. Individual age is often surprisingly hard to determine for individuals in large experimental populations and is frequently impossible to measure non-destructively in field populations. By contrast, suitably chosen measures of development, such as weight or length, are often readily estimated even in quite difficult circumstances. For purposes of comparison with data it is helpful to re-express our description of the equilibrium state in terms of an age-atdevelopment function $\alpha(q)$, and a number-at-development distribution $F(q)$, defined such that $F(q) d q$ is the number of individuals with development in the range $q$ to $q+d q$.

The age-at-development function $\alpha(q)$ and the development-at-age function $\theta(a)$ are related by

$$
\theta(\alpha(q))=q
$$


and the equilibrium number-at-age and number-at-development distributions obey the requirement that

$$
F(q)=\frac{f(\alpha(q))}{g\left(\alpha(q), q, \psi^{*}\right)}
$$

Observable demographic characteristics. Although the operation of the feedback term exemplified by Eq. (4) will in general depend on the shape of the equilibrium distribution, the survival function, $S\left(a, \psi^{*}\right)$, which determines this shape is not itself influenced by the feedback process. Thus the shape of the equilibrium distribution, and all the observable population level demographic characteristics which depend on it can be determined from the demographic characteristics of the constituent individuals without consideration of the feedback mechanisms which produce the environmental configuration needed to establish equilibrium. In the particular case where the onset of reproductive maturity requires the attainment of some critical value of development $\left(q_{M}\right)$ we can see that the age at maturity $(\tau)$, the average immature death rate $(\bar{\delta})$, the average adult fecundity $(\bar{\beta})$, and the ratio of juvenile to adult population $\left(N_{j} / N_{A}\right)$ are

$$
\begin{aligned}
& \tau=\alpha\left(q_{M}\right), \\
& \bar{\delta}=-\frac{1}{\tau} \ln \left[S\left(\alpha\left(q_{M}\right), \psi^{*}\right)\right], \\
& \bar{\beta}=\frac{\int_{\tau}^{\infty} \beta\left(x, \theta(x), \psi^{*}\right) S\left(x, \psi^{*}\right) d x}{\int_{\tau}^{\infty} S\left(x, \psi^{*}\right) d x},
\end{aligned}
$$

and

$$
\frac{N_{J}}{N_{A}}=\frac{\int_{0}^{\tau} S\left(x, \psi^{*}\right) d x}{\int_{\tau}^{\infty} S\left(x, \psi^{*}\right) d x} .
$$

The only unknown in Eqs. (7)-(10) is the equilibrium environment $\psi^{*}$. Once this has been found from Eq. (3b) we can infer the equilibrium values of average fecundity, mortality, development time, and juvenile to adult ratio. However, since we can, in principal, eliminate $\psi^{*}$ between any pair of equations in the group, an alternative view is that Eqs. (7)-(10) define a set of relationships between these observable demographic quantities. Because these relationships are derived by eliminating the equilibrium environment parameter, they are independent of the feedback mechanism by which the environmental equilibrium is established and depend only on the biology of the individuals who comprise the population. For the remainder of this paper we shall thus assume that an equilibrium state is established 
by the operation of an appropriate feedback mechanism without placing any further restriction on the nature or dynamic properties of that feedback mechanism.

\section{Modelling Growth AND FeCUndity}

The growth and fecundity of a food-limited organism are dependent on the rate at which food can be assimilated and on how assimilated material is apportioned between growth, costs, and reproduction. To see how model properties are related to structural assumptions about this partitioning we shall examine a group of models, all of which identify age $(a)$ and weight $(w)$ as the defining physiological variables. For all the models we can relate the development (growth) rate, $g(a, w, \psi)$, to the assimilation rate $(A)$, the maintenance cost rate $(M)$, and the cost in assimilated material of producing a single offspring $\left(W_{E}\right)$ by

$$
g(a, w, \psi) \equiv \frac{d w}{d a}=A(a, w, \psi)-M(a, w, \psi)-w_{E} \beta(a, w, \psi) .
$$

As a simplifying assumption, we regard the material cost of an offspring, $w_{E}$, as constant, and represent it by a single model parameter. All the models we shall investigate assume that individuals feed on a single source of food (density $F$ ) according to a simple type II functional response with a weight-independent half saturation concentration $F_{H}$. Since they all further assume an allometric relationship, $A_{\mathrm{MAX}}=\xi w^{p}$, between maximum assimilation rate and body weight, we select as our measure of individual environment

$$
\psi \equiv \frac{\xi F}{F+F_{H}},
$$

so that $A(a, w, \psi)=\psi w^{P}$.

To complete our model we must specify the cost and fecundity functions $M(a, w, \psi)$ and $\beta(a, w, \psi)$. We follow a number of other workers (Gurney et al, 1990; McCauley et al., 1990b; Kooijman, 1993) in assuming that the basal component of metabolic costs scales directly with weight. There is debate about the possible rules which govern the division between growth and reproduction of any assimilate not required to meet basal costs. We shall therefore explore the demographic implications of four models based on contrasting views of this allocation process.

The first, which we shall refer to as the Kooijman-Metz $(\mathrm{K}-\mathrm{M})$ model, was proposed by Kooijman (1986) to explain von Bertalanffy growth patterns in Daphnia. Its key assumptions are 
- maximum assimilation rate scales with weight to the power 2/3;

- a fraction $\kappa$ of gross assimilate is used for the combination of growth and maintenance, the remainder being used for reproduction in mature $\left(w>w_{M}\right)$ animals and for building gonads in immature animals.

A variant on this model, proposed by Thieme (1988) and hereafter refered to as the Kooijman-Thieme $(\mathrm{K}-\mathrm{T})$ model, assumes that the maintenance of gonads beyond maturity requires a fixed expenditure $C_{M}$, which is charged against the allocation to reproduction. Although this model and the Kooijman-Metz model differ only in their predictions for the resource dependence of fecundity under low-resource conditions, our investigations will show that they predict dramatically different equilibrium demography.

The next allocation model, which we have adapted from the work of Paloheimo et al. (1982) and which we refer to as the Paloheimo (PCT) model, assumes that

- maximum assimilation rate scales with weight;

- mature animals allocate a linearly increasing proportion of net assimilate (after payment of maintenance costs) to reproduction.

It is immediately evident from the second assumption above that, in contrast to both the Kooijman models, the PCT model must predict a resource-independent asymptotic length. To help elucidate the role this feature plays in population demography we have also investigated a fourth model which shares all the features of the PCT model, except that the allocation to reproduction is modified to allow it to predict a resourcedependent asymptotic length. The critical assumptions of this model, which we refer to as the weight-dependent-fecundity (WDF) model are

- maximum assimilation rate scales with weight;

- mature animals have a fecundity linearly related to the amount by which their weight exceeds that at which they become reproductively mature $\left(w_{M}\right)$.

The growth, maintenance, and fecundity rates implied by these four allocation models are shown in algebraic form in Table I. The corresponding age-at-weight and weight-at-age functions are given in Table II. For bookkeeping purposes our statement of the $\mathrm{K}-\mathrm{M}$ and $\mathrm{K}-\mathrm{T}$ models regards assimilate allocated to gonads by immature animals as part of maintenance costs. However, this assumption has no demographic implications at either the individual or the population level. It should be noted that we have stated the minimal set of assumptions which define the behaviour of each model at equilibrium and that additional assumptions are needed in each case to define what happens when some nonequilibrium event, such as a rapid reduction in food supply, occurs. 


\section{TABLE I}

Four Models of Energy Allocation

Kooijman-Metz and Kooijman-Thieme models

Assimilation:

$$
A(a, w, \psi)=\psi w^{2 / 3}
$$

Maintance:

$$
M(a, w, \psi)= \begin{cases}m w+(1-\kappa) \psi w^{2 / 3} & w<w_{M} \\ m w & w \geqslant w_{M}\end{cases}
$$

Fecundity: $\quad \beta(a, w, \psi) \equiv \begin{cases}0 & w \leqslant w_{M} \\ \frac{1}{w_{E}}\left[(1-\kappa) \psi w^{2 / 3}-C_{M}\right] & w \geqslant w_{M}\end{cases}$

Maturity cost: $\quad C_{M} \equiv \begin{cases}0 & \text { Kooijman-Metz } \\ \left(\kappa^{-1}-1\right) m w_{M} & \text { Kooijman-Thieme }\end{cases}$

\section{Weight-dependent fecundity and Paloheimo models}

Assimilation:

$$
\begin{aligned}
& A(a, w, \psi) \equiv \psi w \\
& M(a, w, \psi) \equiv m w
\end{aligned}
$$

Maintenance:

Fecundity: $\quad \beta(a, w, \psi) \equiv \begin{cases}\frac{\eta}{w_{E}}\left(w-w_{M}\right)^{+} & \text {WDF } \\ \frac{\chi}{w_{E}}\left(w-w_{M}\right)^{+}(\psi-m)^{+} w & \text { Paloheimo }\end{cases}$

Note. These models are specified in a minimal form suitable for the calculation of equilibrium states. Additional assumptions are required in nonequilibrium situations.

All four allocation models imply superficially similar, sigmodial, growth curves under constant food conditions (Fig. 1). In each case the weight approaches an asymptotic limit

$$
w_{\infty}= \begin{cases}\left(\frac{\kappa \psi}{m}\right)^{3} & \mathrm{~K}-\mathrm{M} \text { and } \mathrm{K}-\mathrm{T} \text { models } \\ {\left[\frac{\eta}{m+\eta-\psi}\right] w_{M}} & \text { WDF model } \\ w_{M}+\frac{1}{\chi} & \text { PCT model. }\end{cases}
$$

The asymptotic weight in the Kooijman-Metz and Kooijman-Thieme models, whose origin lies in costs rising faster than maximum uptake rate as weight increases, is a very sensitive function of realised assimilation rate, $\psi$. By contrast, the Paloheimo model envisages a constant relationship 


\section{TABLE II}

Weight-at-Age and Age-at-Weight Functions

\section{Kooijman-Metz and Kooijman-Thieme Models}

Age-at-weight:

$$
\alpha(w)=\frac{3}{m} \ln \left[\frac{\kappa \psi-m w_{R}^{1 / 3}}{\kappa \psi-m w^{1 / 3}}\right]
$$

Weight-at-age:

$$
\theta(a)=\left[\frac{\kappa \psi}{m}-\left(\frac{\kappa \psi}{m}-w_{R}^{1 / 3}\right) \exp \left(-\frac{m a}{3}\right)\right]^{3}
$$

Weight-dependent fecundity model

Age-at-weight:

$$
\alpha(w)= \begin{cases}\frac{1}{\psi-m} \ln \left(\frac{w}{w_{R}}\right) & w \leqslant w_{M} \\ a_{M}+\frac{1}{\eta+m-\psi} \ln \left(\frac{(\psi-m) w_{M}}{\eta w_{M}-(\eta+m-\psi) w}\right) & w_{M}<w \leqslant w_{\infty}\end{cases}
$$

Weight-at-age:

$$
\theta(a)= \begin{cases}w_{R} \exp ((\psi-m) a) & a \leqslant a_{M} \\ w_{\infty}\left(1-\frac{\psi-m}{\eta} \exp \left(-(\eta+m-\psi)\left(a-a_{M}\right)\right)\right) & a>a_{M}\end{cases}
$$

where

$$
a_{M} \equiv \frac{1}{\psi-m} \ln \left(\frac{w_{M}}{w_{R}}\right) ; \quad W_{\infty} \equiv \frac{\eta w_{M}}{\eta+m-\psi}
$$

\section{Paloheimo model}

Age-at-weight:

$$
\alpha(w)= \begin{cases}\frac{1}{\psi-m} \ln \left(\frac{w}{w_{R}}\right) & w \leqslant w_{M} \\ a_{M}+\frac{1}{(\psi-m)\left(1+\chi \psi_{M}\right)} \ln \left(\frac{w}{w_{M}\left(1-\chi\left(w-w_{M}\right)\right)}\right) & w>w_{M}\end{cases}
$$

Weight-at-age:

$$
\theta(a)= \begin{cases}w R \exp ((\psi-m) a) & a \leqslant a_{M} \\ \frac{Z w_{M}\left(1+\chi w_{M}\right)}{1+\chi Z w_{M}} & a>a_{M}\end{cases}
$$

where

$$
a_{M} \equiv \frac{1}{\psi-m} \ln \left(\frac{w_{M}}{w_{R}}\right) ; \quad Z \equiv \exp \left((\psi-m)\left(1+\chi w_{M}\right)\left(a-a_{M}\right)\right)
$$



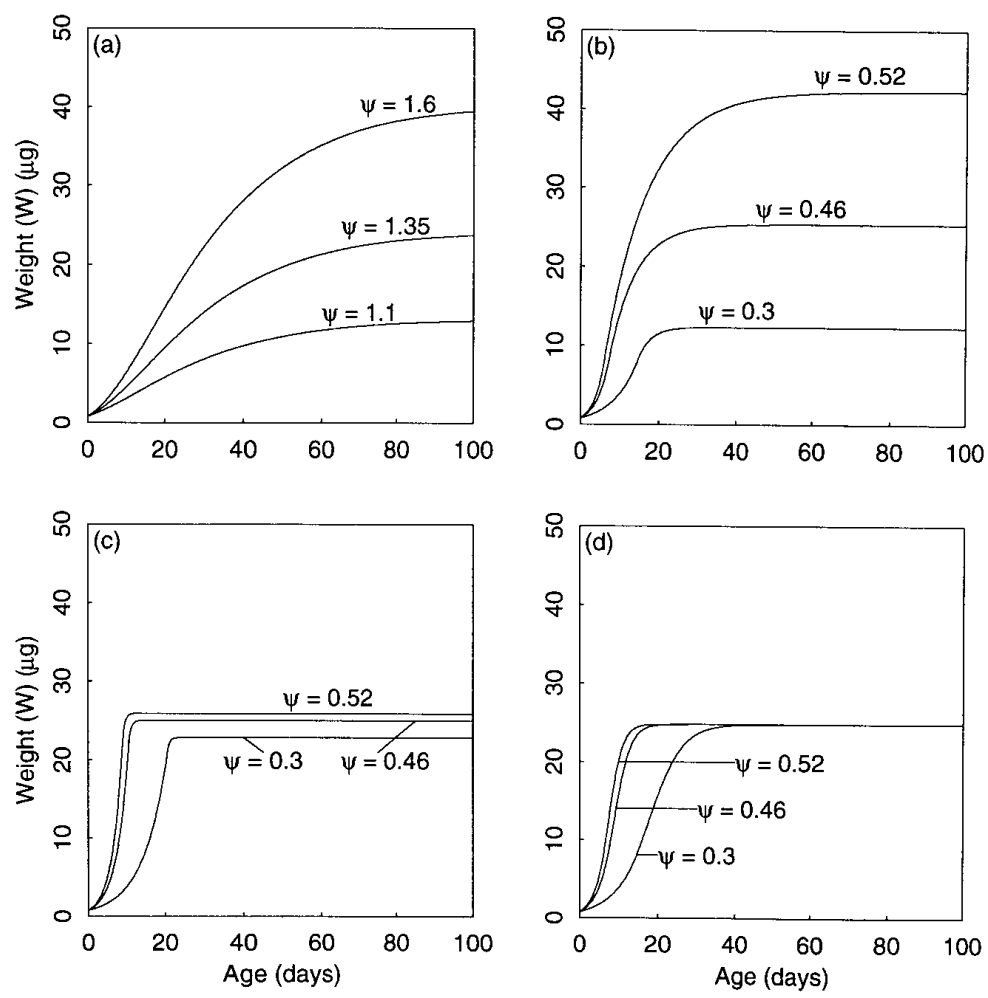

FIG. 1. Growth curves for the four allocation models at different (constant) food densities with Daphnia pulex parameters as given in Table III. (a) Kooijman-Metz and KooijmanThieme models; (b) weight-dependent-fecundity model; (c) weight-dependent-fecundity model with $\eta=2$ days $^{-1}$ and $w_{M}=21 \mu \mathrm{g}$; (d) Paloheimo model.

between costs and uptake, but a switch of net production out of growth and into reproduction which is completed (hence stopping growth) at a food-independent weight. The behaviour of the WDF model lies between these two extremes; the asymptotic weight is always formally dependent on $\psi$, but the sensitivity of this dependence falls as the slope of the fecundity against size curve $(\eta)$ rises.

\section{Modelling MoRtality}

There is a considerable body of experimental observations concerning the energetics of growth and fecundity, and the literature contains a 
number of quite detailed mechanistic models, many of which have been subjected to a variety of comparisons with data. By contrast observations of mortality accompanied by energetic information, even under laboratory conditions, are much less extensive. Models are consequently fewer and much less rigorously tested.

It seems likely that real mortality functions are strongly age and size dependent, with the nature of that dependence often bearing a marked relationship to the individual's energetic status. However, since we have neither tested models nor general principles to guide the formulation of a strategic model of such dependency, we choose instead to consider three rather simple models.

The simplest possible mortality model is a constant per capita mortality independent of both age and weight. There are no circumstances in which such a model is wholly justified, not least because it implies no finite limit to the age to which individuals can live. However, it is a very common modelling assumption where hard information is lacking. Because such an assumption is least indefensible where the prime source of mortality is extrinsic (e.g., predation or environmental stress) we shall refer to this mortality as the background mortality $\delta_{B}$ and write

$$
\delta(a, w, \psi)=\delta_{B}
$$

Survival curves, even under constant food conditions, are seldom if ever exponential, but show decreasing survival per unit time as age increases. We shall investigate the effects of this phenomenon using a linear agedependent mortality model, which postulates that per capita mortality increases linearly above the environmentally defined background level once some threshold $\left(a_{0}\right)$ has been passed; that is, we write

$$
\delta(a, w, \psi)=\delta_{B}\left[1+\lambda\left(a-a_{0}\right)^{+}\right] .
$$

Where food is scarce, mortality is likely to depend on food supply. Over a range of sizes we argue that the best measure of the intensity of such effects is the ratio of assimilation rate over basal metabolic costs. We assume that the approach of this ratio to some threshold $(\rho)$ indicates the onset of starvation conditions and hence of very high mortality. Thus, recalling that in all model variants we assume basal metabolic costs scale linearly with weight, we write

$$
\delta(a, w, \psi)=\delta_{B}\left[1+\frac{\gamma}{(A(w, \psi) / m w)-\rho}\right] .
$$




\section{NUMERICAL INVESTIGATIONS}

Methodology. For some of the models which we shall consider in this paper, it is possible to make analytic calculations of the interrelations between demographic parameters and environment. In some cases it is even possible to derive an analytic expression for the integral in the characteristic Eq. (3b). However, in no case we have studied has it been possible to obtain a closed form solution for the equilibrium value of the environment parameter. We have therefore adopted a uniformly numerical approach to all the models studied, using standard commercially available procedures published by the Numerical Algorithms Group, Ltd. NAG (1994). We used the routine D01AJF to evaluate the finite integral in the survival function (Eq. (2b)) followed by routine D01AMF to evaluate the semi-infinite integral in the characteristic equation (3b). Finally we used the zero-finding routine $\mathrm{C} 05 \mathrm{ADF}$ to determine the equilibrium value of the environment parameter $\left(\psi^{*}\right)$ and hence the stable age distribution and weight-at-age function. From these distributions we inferred the average demographic characteristics defined by Eqs. (7)-(10).
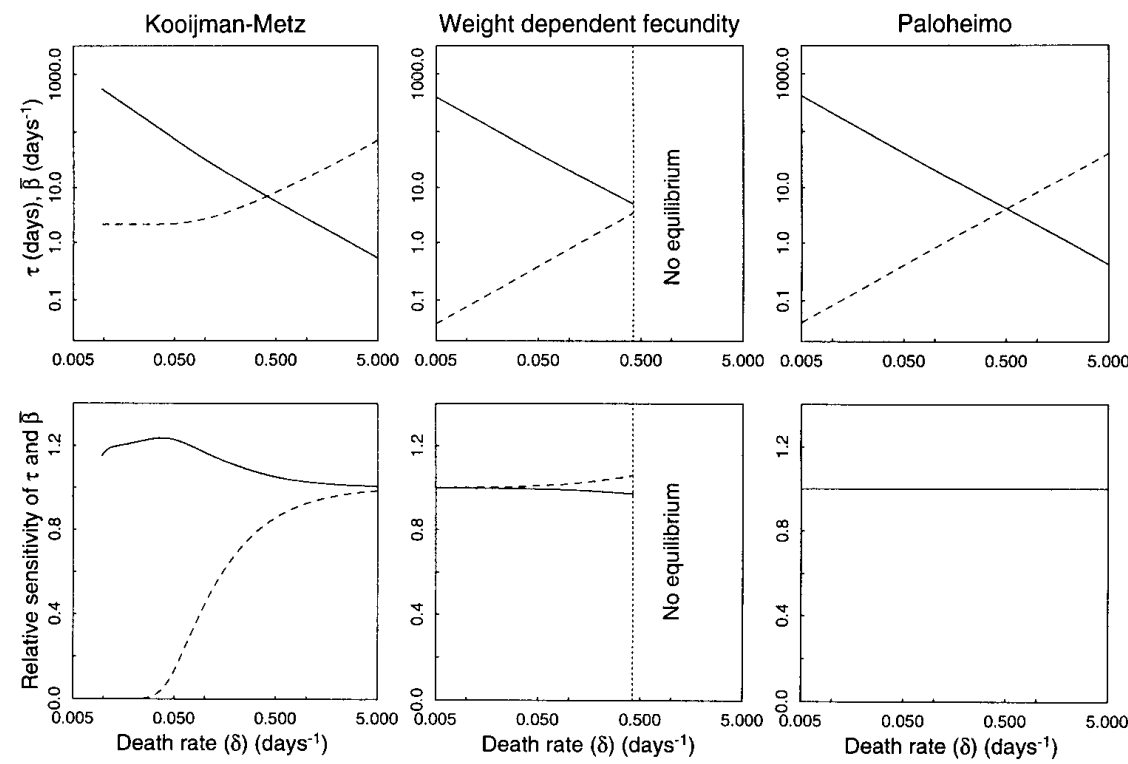

FIG. 2. Observable demographic characteristics of the equilibrium state for four allocation models with density-, age-, and size-independent mortality. Parameters for D. pulex are as given in Table III. Top row: Time to maturity [continuous] and average adult fecundity [dashed]. Bottom row: relative sensitivity in average adult fecundity [dashed] and the negative of the relative sensitivity in time to maturity [continuous]. 
TABLE III

Daphnia pulex Parameters

\begin{tabular}{|c|c|c|}
\hline \multirow[t]{4}{*}{ All models: } & $\Rightarrow w_{R}=0.77 \mu \mathrm{g}$ & $(\Rightarrow$ length at recruitment $=0.6 \mathrm{~mm})$ \\
\hline & $w_{M}=8.1 \mu \mathrm{g}$ & $(\Rightarrow$ length at maturity $=1.6 \mathrm{~mm})$ \\
\hline & $w_{E}=1.23 \mu \mathrm{g}$ & ( $\Rightarrow$ egg wt $=1.6 \times$ weight of recruit $)$ \\
\hline & $m=0.14$ days $^{-1}$ & $(\Rightarrow$ starvation time $=7$ days $)$ \\
\hline Kooijman models: & $\kappa=0.3$ & \\
\hline $\begin{array}{l}\text { Weight-dependent } \\
\text { fecundity: }\end{array}$ & $\eta=0.47$ days $^{-1}$ & $\left(\Rightarrow w_{\infty}=25 \mu \mathrm{g} @ \psi=0.46\right)$ \\
\hline Paloheimo: & $\chi=0.035 \mu \mathrm{g}^{-1}$ & $\left(\Rightarrow w_{\infty}=25 \mu \mathrm{g}\right)$ \\
\hline
\end{tabular}

Note. This table is adapted from values derived by, and data given in, McCauley et al., 1990. The key property to which each parameter has been matched is given in parentheses beside its value.

For each of the models, we have determined how the observable demographic characteristics of the equilibrium population vary as we change the background death rate. We are concerned with large ranges of both background death rate and demographic parameters and so we have displayed the results as $\log -\log$ plots. In some cases, to clarify the implications of these plots, we also plot their slope against the log of the background death rate. This slope represents the relative sensitivity of the observable concerned, that is, the ratio of the proportional change in the observable quantity to the proportional change in background death rate which causes it. To ensure that our results are not confounded by inappropriate or internally inconsistent parameter choices we have focused our investigation of each model round a complete parameter set derived from the literature for Daphnia pulex - see Table III.

Age- and environment-independent mortality. In this section we consider four models, each based on one of the allocation models discussed above, together with constant (i.e. age-, weight-, and density-independent) mortality. In Fig. 2 we show typical results for these models, using the D. pulex parameter set given in Table III.

Simultaneous examination of Figs. 1 and 2 shows that while the two allocation rules which operate on net production (WDF and PCT) imply very different growth trajectories, they yield essentially similar relationships between background death rate and observable demographic parameters at equilibrium. The only exception to this similarity is that there is a critical value of background death rate above which a population of individuals operating the WDF allocation rule cannot achieve equilibrium. Analytic investigation (see Appendix) reveals that this critical death rate must satisfy

$$
\frac{\delta_{\text {crit }}}{\eta}\left(\ln \left(\frac{w_{M}}{w_{R}}\right)\right)+2 \ln \left(\frac{\delta_{\text {crit }}}{\eta}\right)=\ln \left(\frac{w_{M}}{w_{E}}\right) .
$$


The model based on the Kooijman-Metz allocation rule predicts a very different pattern of variation in observable demographic parameters. As background mortality falls from a value corresponding to a lifetime of 0.2 days, the development time initially rises and the fecundity falls in much the same way as we observe under the PCT and WDF allocation rules. We note that the absolute value of fecundity is considerably higher and the development time correspondingly longer than is predicted by the first two rules because of the reduced fraction of assimilate allocated to juvenile growth by the $\mathrm{K}-\mathrm{M}$ rule. However, as background mortality falls to realistic values (corresponding to lifetimes of 5 days or more) the average adult fecundity becomes essentially constant. In this regime, the asymptotic weight has fallen almost to the maturation weight leading to a fecundity almost indistinguishable from that for newly matured individuals, which we denote by $\beta_{M}$. Equilibrium is established wholly by variation of the juvenile development period, which in this regime is given approximately by

$$
\tau \approx \frac{1}{\delta} \ln \left(\frac{\beta_{M}}{\delta}\right)
$$

The extreme lengthening of the juvenile development period which this phenomenon causes is illustrated by the observation that with $D$. pulex parameters a development period of over 90 days is required to reduce juvenile to adult survival sufficiently to establish equilibrium when $\delta_{B}$ is 0.05 (i.e., mean individual lifetime $=20$ days).

At background death rates above 0.5 days $^{-1}$ the model based on KooijmanThieme allocation predicts essentially the same behaviour as that based on its Kooijman-Metz cousin. However, as background mortality falls its behaviour differs dramatically. Here the reduction of asymptotic length towards the maturation length acts to reduce the adult fecundity at an increasing rate (rather than the decreasing rate implied by $\mathrm{K}-\mathrm{M}$ allocation). This leads to fecundity becoming the dominant factor in the establishment of equilibrium. As a corollary of this effect, we see that the development times predicted by this model at very low background death rates are the lowest of all the four models we have examined.

Age-dependent mortality. Figures 3 and 4 show the behaviour of models whose allocation behaviour is described by the four models introduced above, but which have an age-dependent component of mortality, as defined in Eq. (15). Figure 3 shows the effect of mortality rising moderately steeply from age zero $\left(\lambda=1, a_{0}=0\right)$. For all four allocation models, the variation in average juvenile death rate which now accompanies changes in the juvenile to adult development time plays a significant role in the control process. This produces a significant reduction in the value of equilibrium 

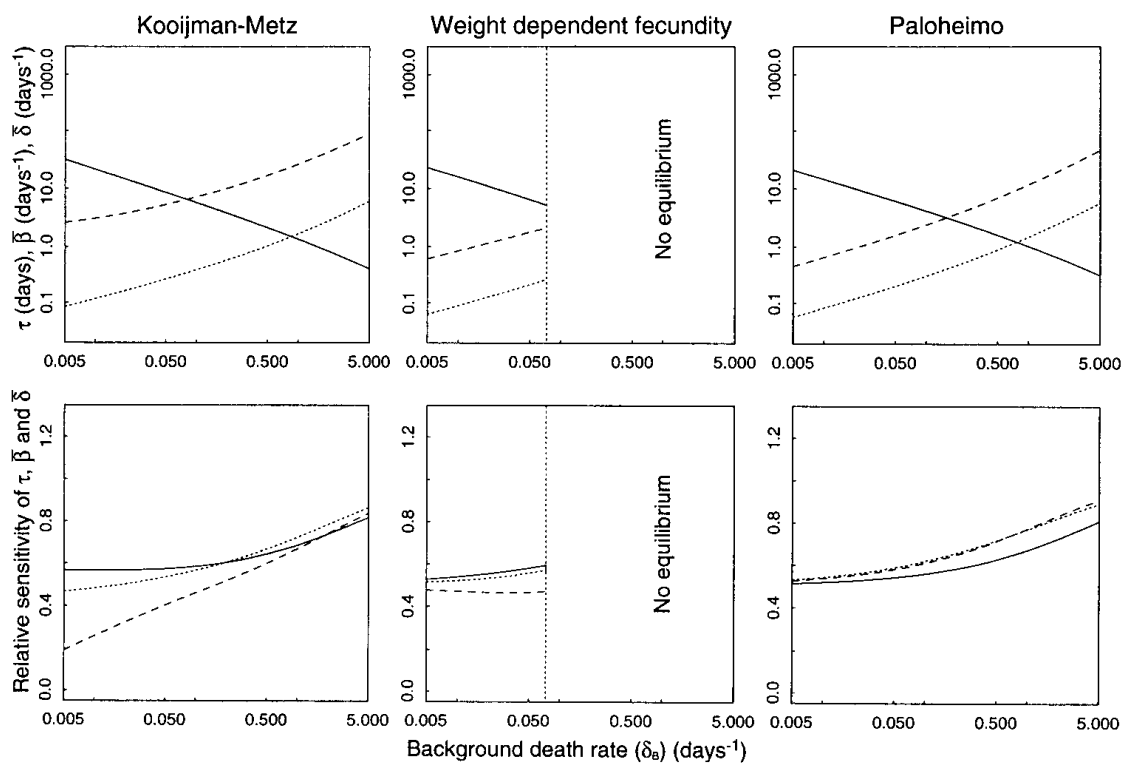

FIG. 3. Observable demographic characteristics of the equilibrium state for four allocation models with linear age-dependent mortality $\delta=\delta_{B}(1+\lambda a), \lambda=1$. Other parameters are as given for $D$. pulex in Table III. Top row: Time to maturity [continuous], average adult fecundity [dashed], and average juvenile mortality [dotted]. Bottom row: Relative sensitivity in average adult fecundity [dashed] and average juvenile mortality [dotted] together with the negative of the relative sensitivity in time to maturity [continuous].

development time and an accompanying increase in equilibrium fecundity compared to the constant mortality case shown in Fig. 2. In the case of the models based on the Kooijman-Metz and Kooijman-Thieme allocation rules we see that these quantitative changes are accompanied by a qualitative change in behaviour. In the constant mortality case, falling background mortality causes either development time (in the $\mathrm{K}-\mathrm{M}$ case) or fecundity (in the $\mathrm{K}-\mathrm{T}$ case) to become the dominant agent of equilibration. In the equivalent cases with linear age-dependent mortality, only the remnants of these effects can be discerned.

Figure 4 shows the effect of an age-dependent mortality mimicking a strong senility effect. In order to exaggerate the effects produced we have set the age of onset of senility at an unrealistically low 10 days $(\lambda=10$, $a_{0}=10$ ). In marked contrast to the constant mortality case, all allocation models now exhibit a low background mortality regime in which adult fecundity varies only slowly with background death rate. That this effect is characteristically different from the behaviour of the Kooijman-Metz variant with constant death rate is made clear by the observation that in 

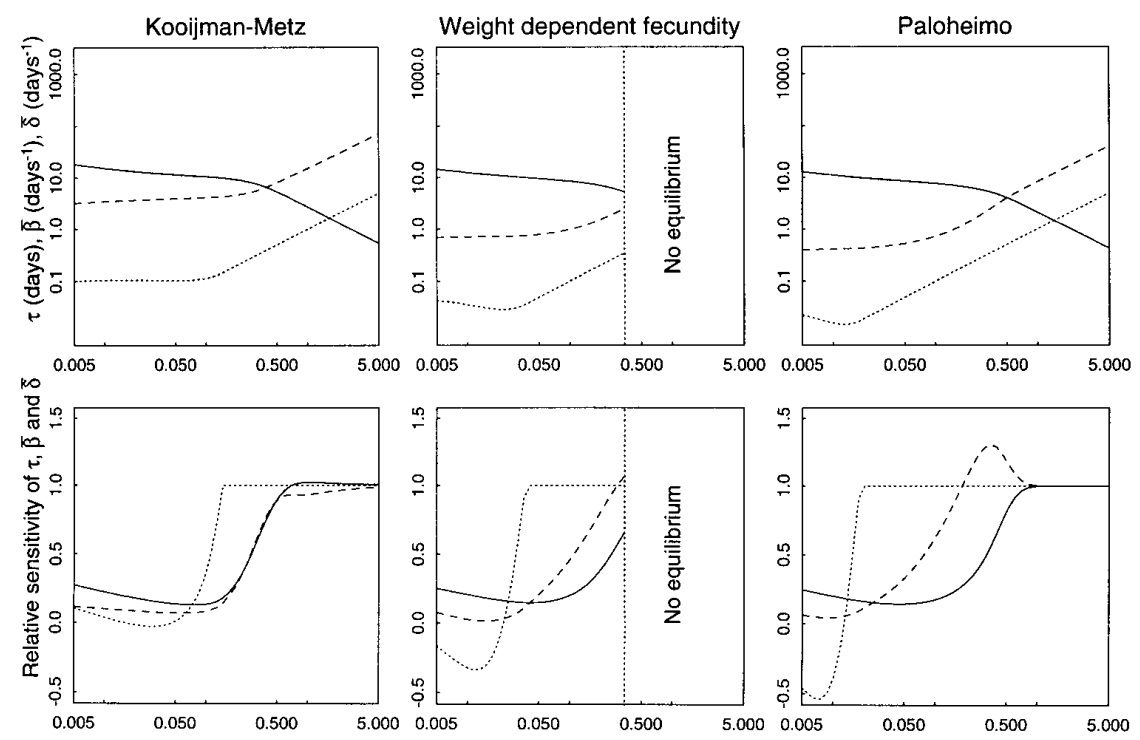

Background death rate $\left(\delta_{\mathrm{e}}\right)\left(\right.$ days $\left.^{-1}\right)$

FIG. 4. Observable demographic characteristics of the equilibrium state for four allocation models with age-dependent mortality strongly focused on old age: $\delta=\delta_{B}\left(1+\lambda\left(a-a_{0}\right)^{+}\right)$, $a_{0}=10, \lambda=10$. Other parameters for $D$. pulex are as given in Table III. Top row: Time to maturity [continuous], average adult fecundity [dashed] and average juvenile death rate [dotted]. Bottom row: Relative sensitivity in average adult fecundity [dashed] and average juvenile mortality [dotted] together with the negative of the relative sensitivity in time to maturity [continuous].

this regime all models show a juvenile delay $(\tau)$ which is only weakly dependent on background mortality.

Because our mortality formulation describes age-dependent mortality as producing a proportional increase over background mortality, the effect we are observing here is not simply due to the age-dependent death rate taking over from the background death rate as the dominant source of mortality. Rather, the system is in a state in which changes in the background mortality are compensated (or even overcompensated) by the change in total mortality induced by small changes in development time. This compensation process is strongly allocation model dependent; leading to an almost perfectly background deathrate-independent regime for the Kooijman-Metz and Kooijman-Thieme variants, but yielding a juvenile mortality which actually falls with increasing background death rate in the other two models. Both these two models (WDF and PCT) show a broadly similar pattern in which nominal control (as measured by relative sensitivity) is exercised by all three demographic characteristics at high background mortality, but is subsequently dominated by fecundity, then juvenile mortality, and lastly delay as background mortality drops. 
Environment-dependent mortality. In Fig. 5 we show how the behaviour of the four models discussed in the first section is affected by the introduction of food-dependent mortality as defined by Eq. (16). As before, the behaviour of the weight-dependent fecundity model is very similar to that of the model based on the Paloheimo allocation rule, except for the region at high background death rates where the WDF model cannot achieve equilibrium. In both cases, a sufficient lowering of background mortality causes the equilibrium environment to fall to the point where the assimilation rate to maintenance ratio approaches the critical value $\rho$. When this happens the density-dependent component of mortality becomes a very sensitive function of environment, and changes in background mortality are accurately compensated for by opposing changes in the densitydependent mortality. The end result is that all the observable demographic characteristics (including the overall average death rate) become almost independent of background mortality.
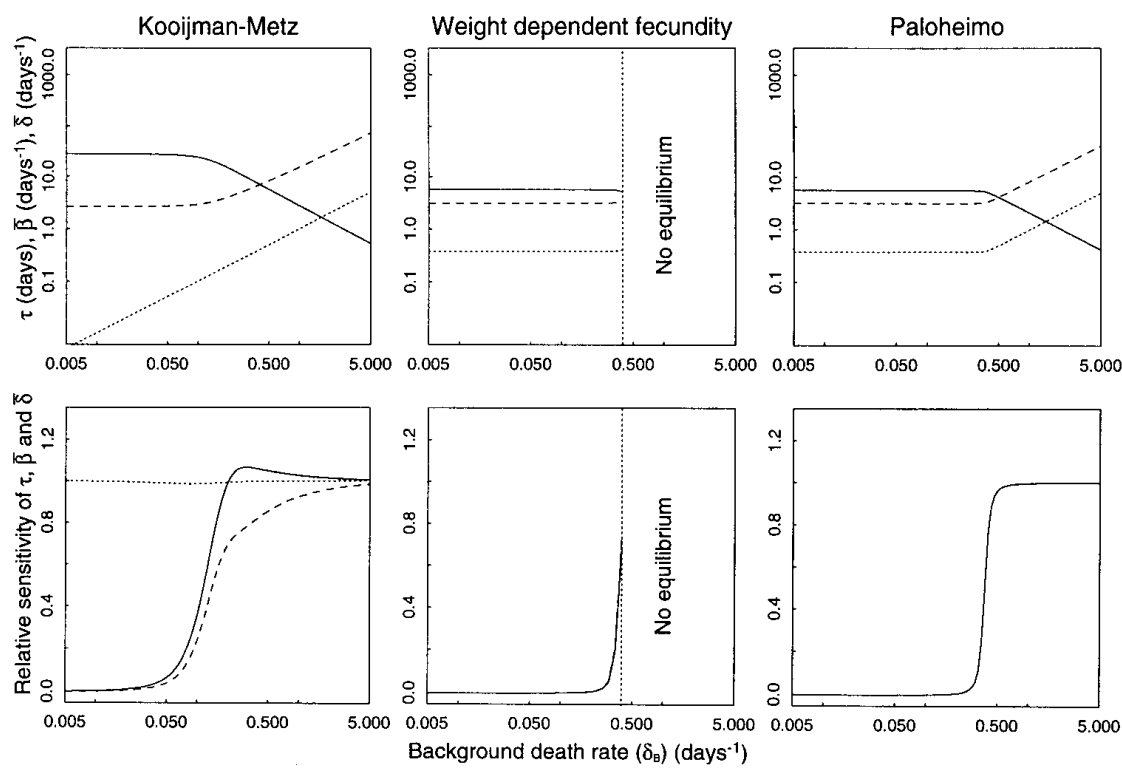

FIG. 5. Observable demographic characteristics of the equilibrium state for four allocation models with strong food-dependent mortality $\delta=\delta_{B}[1+\gamma /(A(w, \psi) / m w-\rho)], \gamma=0.01, \rho=4$. Other parameters for $D$. pulex are as given in Table III. Top row: Time to maturity [continuous], average adult fecundity [dashed], and average juvenile death rate [dotted]. Bottom row: Relative sensitivity in average adult fecundity [dashed] and average juvenile mortality [dotted] together with the negative of the relative sensitivity in time to maturity [continuous]. 
The models based on Kooijman-Metz and Kooijman-Thieme allocation show a low-background mortality regime in which the time to maturity and the average fecundity become essentially constant, but where the average juvenile mortality remains highly sensitive to changes in this background mortality. The reason for this distinctive behaviour is that, in contrast to the WDF and PCT models, the Kooijman-Metz and Kooijman-Thieme models assume that assimilation and maintenance have different allometry. Thus, when assimilation approaches the critical value in these models, it does so only for the larger individuals. This implies that while juvenile mortality remains dominated by background mortality, adult mortality rises strongly as background mortality decreases, leading to a steady shift of mortality pressure away from juveniles and towards adults. Fig. 6 shows the resulting pattern of mortality changes for the Kooijman-Metz model.

Population size structure. In the proceeding three sections, to facilitate the discussion of causal mechanisms, we have concentrated on the behaviour of average values of fecundity, mortality, and development time. However, one of the measures of population demography which is most

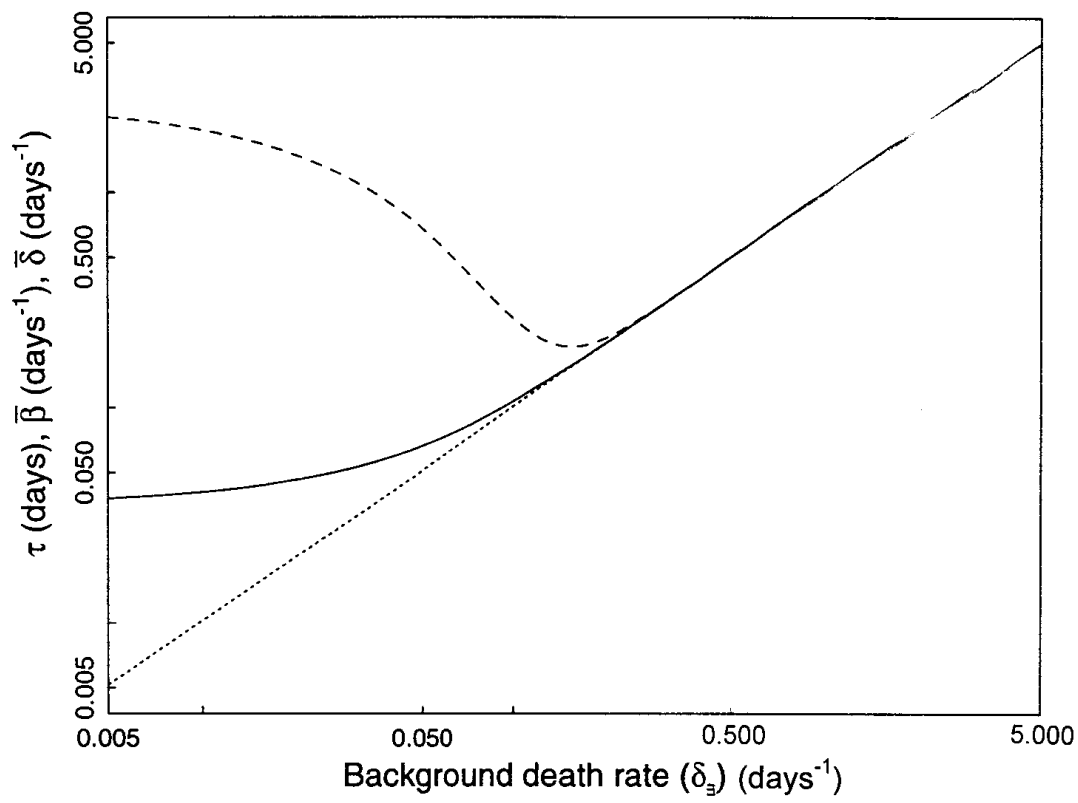

FIG. 6. Changes in juvenile [dotted], adult [dashed], and overall [continuous] mortality for the Kooijman-Metz allocation model with strong food-dependent mortality, $\left.\delta=\delta_{B}[1+\gamma /(A(w, \psi) / m w)-\rho)\right], \gamma=0.01, \rho=4$. Other parameters for D. pulex are as given in Table III. 


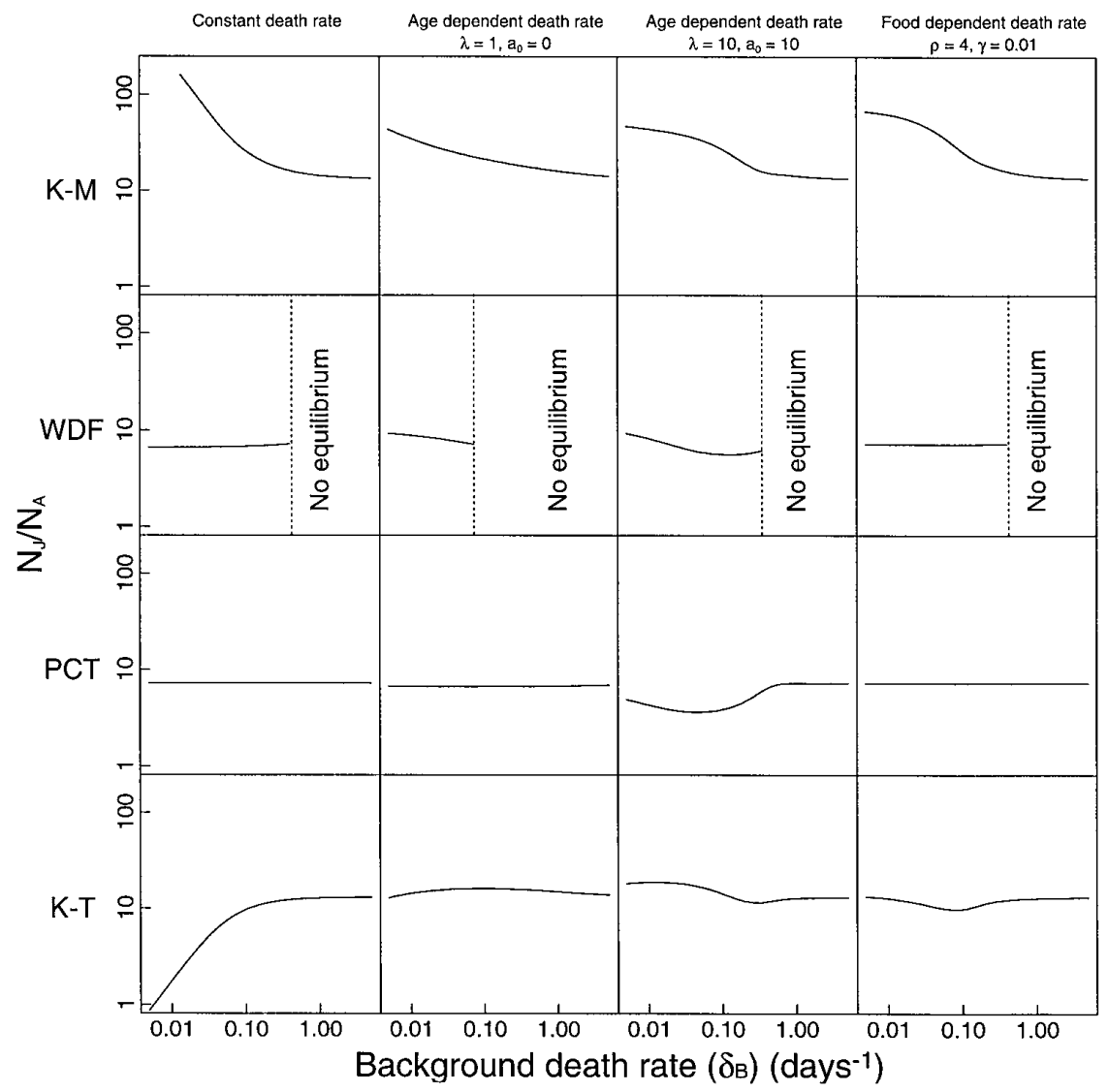

FIG. 7. Juvenile-to-adult ratio as a function of background death rate for four allocation and four death rate models. The rows show, from top to bottom, the variation for the Kooijman-Metz, weight-dependent-fecundity, Paloheimo, and Kooijman-Thieme allocation models. The columns, from left to right, show the variation with constant mortality, linear age-dependent mortality, strong senility, and resource-dependent mortality.

readily observable in the field is the ratio of the abundance of juvenile individuals to that of adults. In Fig. 7 we show the variation of this ratio with changes in background mortality for all the models we have considered. No model based on either WDF or PCT allocation predicts changes in juvenile to adult ratio greater than a factor of two, and in most cases the predicted change is only a few tens of percent. In contrast, all variants of the $\mathrm{K}-\mathrm{M}$ model predict that changing background mortality can induce changes of up to an order of magnitude in $N_{J} / N_{A}$. The model with $\mathrm{K}-\mathrm{T}$ allocation and constant mortality predicts a similar size change in population structure, albeit in the opposite direction. However, the 
remaining models with $\mathrm{K}-\mathrm{T}$ allocation show population structure remaining essentially constant as background mortality changes.

\section{DisCUSSION}

The mechanisms of population control. We have examined models of growth and reproduction coupled with 4 models of mortality to form a total of 16 distinct models of individual demography. The results shown in this paper have all been calculated using a set of parameters (Table III) which are loosely designed to represent $D$. pulex, but we have also conducted wide ranging explorations of the parameter space of an the models and have found the behaviour portrayed here to be typical.

The most consistent pattern is the strong similarity in the control behaviour between the models based on Paloheimo and weight-dependentfecundity allocation. By contrast, the models based on Kooijman-Metz and Kooijman-Thieme allocation predict patterns of variation in control behaviour which can differ strongly both from the WDF and PCT models and from each other. This difference is particularly marked in the case of constant mortality, where the PCT- and WDF-based models show all observable demographic parameters changing together over the entire range of death rates. In contrast, the $\mathrm{K}-\mathrm{M}$ model shows fecundity becoming essentially constant (and hence uninvolved in population control) at low background death rates, while the $\mathrm{K}-\mathrm{T}$ model shows a rapid escalation in the relative importance of fecundity in population control as background death rate falls.

The similarity in control properties between WDF- and PCT-based models occurs despite the fact that the growth curves predicted by the WDF model show strongly food-dependent asymptotic length, while the PCT model growth curves all asymptote at the (food-independent) weight at which proportional allocation to reproduction equals unity. We believe that the key similarities between the two models are, first, that both allocation rules operate on net assimilate and, second, that both models assume the same allometry for costs and maximum assimilation rate.

If the similarity between the PCT- and WDF-based models is due to these two factors we should seek the reasons for the distinctive behaviour of the Kooijman-Metz-based model in these same areas. Close examination of the constant mortality case for the $\mathrm{K}-\mathrm{M}$ model reveals that in the low-death-rate regime the realised assimilation rate falls very close to the point at which maintenance costs are equal to that fraction of gross assimilate allocated to the combination of growth and maintenance. Since maintenance takes priority over growth, the amount of assimilate then available for growth necessarily becomes very small. This implies a very 
small realised growth rate and a commensurately long development time. Under such conditions, a very small proportional change in assimilation rate causes a very large proportional change in development time. However, at the same time, fecundity remains significant and relatively constant. This happens because a fixed fraction of gross assimilate $(70 \%$ in our examples) is being allocated to reproduction, so that changes in assimilation rate are coupled proportionally to changes in fecundity. This clearly implies that the small changes in assimilation needed to compensate for changes in background mortality will be almost exclusively expressed as changes in development time. By contrast, the allocation rules based on net assimilate lead to development time and fecundity being strongly coupled under all conditions.

The explanation for the distinctive behaviour of the $\mathrm{K}-\mathrm{M}$ model under the assumption of an assimilation-rate-dependent mortality lies in a mathematically similar mechanism, this time centred on different assumptions about allometry. The PCT and WDF models both assume that costs and assimilation have the same allometry. Thus the environment-dependent mortality defined by Eq. (16) acts in an age-independent way. However, it is a fundamental requirement for growth curve saturation in the $\mathrm{K}-\mathrm{M}$ model that costs rise faster with weight than assimilation. The inevitable implication of this is that when applied to the $\mathrm{K}-\mathrm{M}$ model, food-dependent mortality acts almost exclusively on the older (i.e., adult) individuals. We see the effect of this illustrated clearly in Fig. 6, where at high background mortality, enhanced mortality is entirely absent and all age classes show total mortality equal to the background level. At low background mortality, however, while juvenile mortality remains at the background level, adult mortality rises strongly, eventually reaching levels which totally compensate for changes in background death rate and hence cause both development time and fecundity to become independent of background mortality.

The Kooijman-Metz and Kooijman-Thieme-based models predict strongly similar behaviour (albeit with fecundity and delay exchanging roles) in all circumstances except constant mortality. This happens because only under constant mortality is the element in which they differ (resource control of fecundity) the main determinant of the changes we observe. As a constant background mortality declines in size both models predict that the equilibrium resource level declines towards a level at which the asymptotic weight approaches the maturation weight. At the same time, the time required to reach maturity increases and the juvenile to adult survival declines. In the $\mathrm{K}-\mathrm{M}$ model this process is accompanied by the fecundity tending to a constant value, so the adjustment of juvenile survival must be very extreme to produce equilibrium. In the case of the $\mathrm{K}-\mathrm{T}$ model the fecundity is roughly proportional to the difference between the asymptotic 
weight and the maturation weight, so as $w_{\infty}$ approaches $w_{M}$ the fecundity drops faster than the juvenile survival and takes over as the main controlling process.

Implications. By examining the variation in the sensitivity of observable demographic parameters to changes in background death rate, we have been able to identify the demographic processes which would be identified observationally as being the dominant controlling influence under any given set of circumstances. However, we have shown that extrapolation of such identification outside the parameter range of observations is likely to prove misleading. Indeed, even within range of observations, safe identification requires very complete information. The contrast between the $\mathrm{K}-\mathrm{M}$ example in Fig. 5 and the more complete information given in Fig. 6 illustrates the potential for mistaken identification of critical process.

This study has also shown that, although the ultimate expression at the population level of assumptions made at individual level is by no means counterintuitive, the linkages can be quite subtle and are therefore relatively hard to predict in the formulation stage of a modelling enterprise. Moreover, we have shown that assumptions often substituted for detailed knowledge in unstructured population modelling (e.g., a constant death rate) are much less innocuous in the context of structured models. This all implies that the information requirements for formulating individual-based models (of any description) must be expected to be rather greater than might be anticipated on the basis of the requirements of unstructured models. It also emphasises the paramount necessity of testing and validation before any model is used for predictive purposes (cf. Murdoch et al., 1992).

Our analysis has shown that different but still plausible assumptions about energy aquisition and allocation can lead to models which predict that any or all of the key demographic properties (fecundity, mortality, or development time) can act as the central agent of population control. However, in real populations there are often significant physiological restraints on the range of plausible values for these variables and in specific cases this may allow us to reject some allocation rules on the grounds that they imply the violation of these constraints. For example, the development time for $D$. pulex probably cannot vary more than about 6-fold, whereas the fecundity and mortality can vary at least 20 -fold and perhaps more (McCauley et al., 1990a). Similarly freshwater fish such as trout must generally mature by their second or third season while being able to vary their food-dependent mortality and fecundity rates by one or two orders of magnitude. A similar conclusion holds for many insects, where larval food limitation is generally expressed as small adult size rather than delayed development, and for marine fish and invertebrates. These considerations 
suggest that for many species, the Kooijman-Metz allocation model may be a less appropriate representation than either the Kooijman-Thieme model or the net assimilate models (PCT and WDF).

\section{Appendix: Characteristic Equation for the WDF Model}

Substitution of the appropriate functional forms and evaluation of the integral in Eq. (3b) shows the characteristic equation for the WDF model can be expressed as

$$
\left(\frac{W_{M}}{W_{R}}\right)^{\delta /(\psi-m)}=\left(\frac{W_{M} \eta}{W_{E} \delta}\right)\left[\frac{\psi-m}{\delta+\eta-\left(\psi_{m}\right)}\right] .
$$

Given that $w_{M}>w_{R}$ the LHS is a monotone decreasing function of $(\psi-m)$ with a singularity at $(\psi-m)=0$. The RHS is a monotone increasing function of $(\psi-m)$ whose value goes to zero with $(\psi-m)$. For a solution with finite value of the asymptotic length we require $(\psi-m)<\eta$. We are guaranteed one and only one such solution iff the value of the LHS at $(\psi-m)=\eta$ is less than the value of the RHS at the same point. Hence, for a viable solution we require

$$
\left(\frac{w_{M}}{w_{R}}\right)^{\delta / \eta}<\left(\frac{w_{M} \eta}{w_{E} \delta}\right)\left(\frac{\eta}{\delta}\right)=\frac{w_{M}}{w_{E}}\left(\frac{\eta}{\delta}\right)^{2} .
$$

\section{ACKNOWLEDGMENTS}

This work was supported by CEC MAST Contract MAS2-CT920033, NSF Grant DEB9319301, and EPA Grant R819433-01-0.

\section{REFERENCES}

Anderson, J. J. 1992. A vitality based stochastic model for organism survival, in "IndividualBased Models and Approaches in Ecology" (DeAngelis and Gross, Eds.), Chapman \& Hall, London/New York.

Broekhuizen, N., Gurney, W. S. C., Jones, A., And Bryant, A. D. 1994. Modelling compensatory growth, Funct. Ecol., to appear.

Gurney, W. S. C., McCauley, E., Nisbet R. M., and Murdoch, W. W. 1990. The physiological ecology of Daphnia: A dynamic model of growth \& reproduction, Ecology 71, 716-732.

Gurtin, M. E., AND MCCAMY, R. C. 1981. Diffusion models for age-structured populations, Math. Biosci. 54, 49-59. 
Huston, M. A. 1992. Individual-based forest succession models and the theory of plant competition, in "Individual-Based Models and Approaches in Ecology" (DeAngelis and Gross, Eds.), Chapman \& Hall, London/New York.

Kooljman, S. A. L. M. 1986. Population dynamics on the basis of budgets, in "The Dynamics of Physiologically Structured Populations" (Metz and Diekmann, Eds.), Springer-Verlag, Berlin/Heidelberg.

Kooljman, S. A. L. M. 1993. "Dynamic Energy Budgets in Biological Systems," Cambridge Univ. Press, Cambridge.

MacKay, N. 1992. Evaluating the size effects of lampreys and their prey: An application of an individual-based model, in "Individual-Based Models and Approaches in Ecology" (DeAngelis and Gross, Eds.), Chapman \& Hall, London/New York.

MAY, R. M. 1981. "Theoretical Ecology: Principals \& Applications," Blackwell, Oxford.

McCauley, E, Murdoch, W. W., And Nisbet, R. M. 1990a. Growth, reproduction and mortality of Daphnia pulex: Life at low food, Funct. Ecol. 4, 505-514.

McCauley, E., Murdoch, W. W., Nisbet, R. M., and Gurney, W. S. C. 1990b. The physiological ecology of Daphnia: Development of a model of growth and reproduction, Ecology 71, 703-715.

Metz, J. A. J., AND Diekmann, O. 1986. "The Dynamics of Physiologically Structured Populations," Springer-Verlag, Berlin/Heidelberg.

Murdoch, W. W., McCauley, E., Nisbet, R. M., Gurney, W. S. C., and de Roos, A. M. 1992. Individual-based models; combining testability and generality, in "Individual-Based Models and Approaches in Ecology" (DeAngelis \& Gross, Eds.), Chapman \& Hall, London/New York.

NAG. 1994. "Nag Fortran Library Mk16," Numerical Algorithms Group, Ltd, Oxford.

Paloheimo, J. E., Crabtree, S. J., and Taylor, W. D. 1982. Growth model of Daphnia, Can. J. Fish. Aq. Sci. 39, 598-606.

Ross, A. H., AND NisBet, R. M. 1990. Dynamic models of growth and reproduction of the mussel Mytilus edulis L., Funct. Ecol. 4, 777-787.

Thieme, H. R. 1988. Well-posedness of physiologically structured population models for Daphnia magna, J. Math. Biol. 26, 299-317. 